\title{
The Enumeration of Sequences with Restrictions on their Partial Sums
}

\author{
Stephen Suen \\ Department of Mathematics and Statistics \\ University of South Florida \\ ssuen@usf .edu
}

\author{
Kevin P. Wagner \\ Department of Mathematics and Statistics \\ University of South Florida \\ kwagner@mail.usf .edu
}

Submitted: Mar 26, 2010; Accepted: Nov 17, 2010; Published: Nov 26, 2010

Mathematics Subject Classification: 05A15, 05A10

\begin{abstract}
We examine sequences containing $p$ " $-t$ "s and $p t+r$ " +1 "s, where $p, t$, and $r$ are integers satisfying $p \geqslant 0, t \geqslant 1$ and $p t+r \geqslant 0$. We develop a rotation method to enumerate the number of sequences meeting additional requirements related to their partial sums. We also define downcrossings about $\ell$ and their downcrossing numbers, and obtain formulas for the number of sequences for which the sum of the downcrossing numbers equals $k$, for $\ell \leqslant r+1$. We finish with an investigation of the first downcrossing number about $\ell$, for any $\ell$.
\end{abstract}

Keywords. Lattice paths, ballot problem, rotation method, crossings, crossing sums, generalized binomial series.

\section{Introduction}

We shall assume throughout that $p$, $t$, and $r$ are integers satisfying $p \geqslant 0, t \geqslant 1$ and $p t+r \geqslant 0$. Let $\Omega=\Omega_{p, r}=\Omega_{p, r}^{(t)}$ denote the collection of all sequences containing $p$ " $-t$ "s and $p t+r$ " +1 "s. For a sequence $\omega \in \Omega$, let $\omega_{j}$ denote its $j^{\text {th }}$ digit, and let $S_{k}(\omega)$ denote its $k^{\text {th }}$ partial sum. That is,

$$
S_{k}=\sum_{j=1}^{k} \omega_{j}, \quad \text { with } S_{0}=0 \text { and } S_{p t+p+r}=r .
$$

One common way to picture the sequences in relation to their partial sums is by considering the sequences as paths $\left\{\left(j, S_{j}\right): 0 \leqslant j \leqslant p t+p+r\right\}$, with each " +1 " meaning "go right one, go up one," and each "- $t$ " meaning "go right one, go down $t$." We study the number of paths with conditions on their partial sums, on their number of crossings, crossing 
numbers, and crossing sums, which we shall define later. Our interest in these sequences originated from our investigation of the acceptance urn model involving $p t+r$ " +1 " balls and $p$ " $-t$ " balls. (For $t=1$, see Chen et. al. [1] and Suen and Wagner [12].) These sequences are also related to lattice paths (see Krattenthaler [5] and Mohanty [7]), the ballot problem (see Renault [9] and Takács [13]), and rank order statistics in generalized random walks (see Saran and Rani [10]).

When $t=1$, the corresponding paths are known as ballot paths, and the study of these paths in $\Omega$ often uses the reflection principle (see for example Feller [2]). When $t \geqslant 2$, the reflection principle no longer applies, and the idea of rotation is used instead (see for example Goulden and Serrano [3]). To describe the idea of rotation, we denote by $\omega(i, j]$, for $\omega \in \Omega$, the sequence obtained by reversing the $(i+1)^{\text {th }}$ to $j^{\text {th }}$ digits of $\omega$. In particular, we denote $\omega^{R}$ as the reversal of $\omega$, that is, $\omega^{R}=\omega(0, p t+p+r]$. Clearly, $\omega(i, j] \in \Omega$ for any $i<j$.

Lemma 1.1 (The Reversal Lemma). We have $S_{n}(\omega)=S_{n}(\omega(i, j])$ for $n \leqslant i$ and $n \geqslant j$, and $S_{n}(\omega(i, j])+S_{i+j-n}(\omega)=S_{j}(\omega)+S_{i}(\omega)$ for $i \leqslant n \leqslant j$.

Proof. Observe that for $i \leqslant n \leqslant j$, we have

$$
S_{n}(\omega(i, j])=S_{i}(\omega)+\sum_{\ell=j-n+1}^{j} \omega_{\ell}, \quad S_{i+j-n}(\omega)=S_{i}(\omega)+\sum_{\ell=i+1}^{j-n} \omega_{\ell} .
$$

Thus,

$$
S_{n}(\omega(i, j])+S_{i+j-n}(\omega)=2 S_{i}(\omega)+\sum_{\ell=i+1}^{j} \omega_{\ell}=S_{i}(\omega)+S_{j}(\omega) .
$$

From a path perspective, the map from $\omega$ to $\omega(i, j]$ rotates the portion of the path $\omega$ over $[i, j]$ by 180 degrees about the point $P=\left((i+j) / 2,\left(S_{i}+S_{j}\right) / 2\right)$, while keeping the rest of the path intact. (See Figure 1 for an example.) In this regard, Lemma 1.1 can also be called the Rotation Lemma. This transformation can also be described as a reflection, vertically and horizontally, of the part of $\omega$ over $[i, j]$ through the midpoint $P$, a notion which also holds in higher dimensions. Thus, the Reversal Lemma gives rise to a midpoint reflection method.

Let $R_{p, r}(\ell)$ denote the number of paths in $\Omega_{p, r}$ with all partial sums at most $\ell$. That is, $R_{p, r}(\ell)$ is the number of $\omega \in \Omega$ for which $S_{j}(\omega) \leqslant \ell$ for all $j$ satisfying $0 \leqslant j \leqslant p t+p+r$. Similarly, let $R_{p, r}^{\prime}(\ell)$ denote the number of paths with all partial sums at least $\ell$. The following is an immediate consequence of the Reversal Lemma.

Corollary 1.2. For any integer $\ell, R_{p, r}^{\prime}(r-\ell)=R_{p, r}(\ell)$.

Proof. Apply the Reversal Lemma to the entire sequence. Then for each $\omega \in \Omega$, we have for all $j$ that

$$
S_{j}\left(\omega^{R}\right)=S_{0}(\omega)+S_{p t+p+r}(\omega)-S_{p t+p+r-j}(\omega)=r-S_{p t+p+r-j}(\omega) .
$$

That is, $S_{j}(\omega) \leqslant \ell$ for all $j$ if and only if $S_{j}\left(\omega^{R}\right) \geqslant r-\ell$ for all $j$. Since the map $\omega \rightarrow \omega^{R}$ is a bijection, the result now follows. 
We shall need the following tools for the ease of discussion that follows. If $S_{n}(\omega)=a$, $S_{q}(\omega)=b$, and $S_{k}(\omega)<b$ for $n \leqslant k<q$, we say that the path has made a " $+(b-a)$ " trip. Similarly, if $S_{n}(\omega)=a, S_{q}(\omega)=b$, and $S_{k}(\omega)>a$ for $n<k \leqslant q$, we say that the path has made a reverse " $+(b-a)$ " trip. (A " +0 " trip is the empty path.) Note that, upon reversal, a reverse " $+\ell$ " trip becomes a " $+\ell$ " trip, and vice versa. A path in $\Omega_{p, 0}$ with nonnegative partial sums is called a Dyck path. We allow $p=0$ in which case we have an empty Dyck path. We shall say that a nonempty Dyck path is strict if $S_{j} \geqslant 1$ for all $j \neq 0, p t+p$. A reverse Dyck path is one whose reversal is a Dyck path (i.e. $S_{j} \leqslant 0$ for all $j$ ), and a strict reverse Dyck path is similarly defined.

The paths in $\Omega$ have been discussed in Graham, Knuth and Patashnik [4]. We shall give a brief account of what is known or easily deduced. For each positive integer $t$, let

$$
B_{p, r}^{(t)}=\left(\begin{array}{c}
p t+p+r \\
p
\end{array}\right), \quad \text { and } \quad C_{p, r}^{(t)}=\left(\begin{array}{c}
p t+p+r \\
p
\end{array}\right) \frac{r}{p t+p+r}
$$

with $C_{p, 0}^{(t)}=\delta_{p, 0}$, where $\delta$ is Kronecker's delta. We shall leave most of our results in terms of these coefficients. Then $\left|\Omega_{p, r}\right|=B_{p, r}^{(t)}$. When $r=1$, the sequences in $\Omega$ are known as Raney sequences, and the numbers $C_{p, 1}^{(t)}$ are Fuss-Catalan numbers. (When $t=r=1$, see Stanley [11] for the many different interpretations of the numbers $C_{p, 1}^{(1)}=\frac{1}{2 p+1}\left(\begin{array}{c}2 p+1 \\ p\end{array}\right)$.) In this paper, since $t$ is fixed, we will omit the superscripts, using the simplified notation $B_{p, r}$ and $C_{p, r}$ instead. It is known (see for example [4]) that $C_{p, 1}$ is the number of Raney sequences in $\Omega_{p, 1}$ with $S_{j} \geqslant 1$ for all $j \geqslant 1$. Since the first element of these paths is always a " +1 ," the number of paths in $\Omega_{p, 0}$ with nonnegative partial sums also equals $C_{p, 1}$. That is, the numbers of Dyck paths and reverse Dyck paths in $\Omega_{p, 0}$, using Corollary 1.2, equal

$$
R_{p, 0}(0)=R_{p, 0}^{\prime}(0)=C_{p, 1} \text {. }
$$

Since a " +1 " trip is composed of a reverse Dyck path followed by a " +1 ," the number of paths in $\Omega_{p, 1}$ that are themselves " +1 " trips equals $C_{p, 1}$.

Note that a nonempty Dyck path must end with a "-t." This Dyck path, with the final " $-t$ " removed, can be decomposed into $t+1$ Dyck paths, with consecutive Dyck paths separated by a " +1 ." This shows that for $p \geqslant 1$,

$$
C_{p, 1}=\sum_{\substack{p_{i} \geqslant 0 \\ p_{1}+p_{2}+\cdots+p_{t+1}=p-1}} C_{p_{1}, 1} C_{p_{2}, 1} \cdots C_{p_{t+1}, 1}
$$

If we define, following [4],

$$
\mathcal{B}_{t}(z)=\sum_{p \geqslant 0} \frac{1}{p t+1}\left(\begin{array}{c}
p t+1 \\
p
\end{array}\right) z^{p}
$$

then

$$
\mathcal{B}_{t+1}(z)=\sum_{p \geqslant 0} C_{p, 1} z^{p}, \quad \text { and from }(1), \quad \mathcal{B}_{t+1}(z)=z \mathcal{B}_{t+1}(z)^{t+1}+1
$$


Lagrange's inversion now gives

$$
\mathcal{B}_{t+1}(z)^{r}=\sum_{p \geqslant 0} C_{p, r} z^{p}
$$

and

$$
\frac{\mathcal{B}_{t+1}(z)^{r}}{1-z(t+1) \mathcal{B}_{t+1}(z)^{t}}=\sum_{p \geqslant 0} B_{p, r} z^{p} .
$$

For integer $r>0$, the number of paths in $\Omega_{p, r}$ that are themselves " $+r$ " trips equals

$$
\sum_{\substack{p_{i} \geqslant 0 \\ p_{1}+p_{2}+\cdots+p_{r}=p}} C_{p_{1}, 1} C_{p_{2}, 1} \cdots C_{p_{r}, 1}=\left[z^{p}\right] B_{t+1}(z)^{r}=C_{p, r}
$$

where $\left[z^{p}\right] G(z)$ denotes the coefficient of $z^{p}$ in $G(z)$. More generally, the convolution of " $+r_{i}$ " trips, where $r_{i} \geqslant 1$ and $1 \leqslant i \leqslant k$, gives

$$
\sum_{\substack{p_{i} \geqslant 0 \\ p_{1}+p_{2}+\cdots+p_{k}=p}} C_{p_{1}, r_{1}} C_{p_{2}, r_{2}} \cdots C_{p_{k}, r_{k}}=C_{p, r_{1}+r_{2}+\cdots+r_{k}} .
$$

In addition, using (2) and (3), we have

$$
B_{p, r+s}=\left[z^{p}\right] \frac{\mathcal{B}_{t+1}(z)^{r+s}}{1-z(t+1) \mathcal{B}_{t+1}(z)^{t}}=\sum_{k=0}^{p} C_{p-k, r} B_{k, s} .
$$

Finally, each strict reverse Dyck path in $\Omega_{p, 0}$, where $p \geqslant 1$, consists of a " $t$ " followed by a " $+t$ " trip. With the " $t$ " removed, they are paths in $\Omega_{p-1, t}$ that are themselves " $+t$ " trips. Therefore, the number of strict Dyck paths (or strict reverse Dyck paths) in $\Omega_{p, 0}$ equals $C_{p-1, t}$. We have thus proved the following theorem.

Theorem 1.3. (a) For integer $r>0$, the number of paths in $\Omega_{p, r}$ that are " $+r$ " trips (or reverse " $+r$ " trips) equals $C_{p, r}$.

(b) The number of Dyck paths (or reverse Dyck paths) in $\Omega_{p, 0}$ equals $C_{p, 1}$.

(c) The number of strict Dyck paths (or strict reverse Dyck paths) in $\Omega_{p, 0}$ equals $C_{p-1, t}$.

Recall that $R_{p, r}(\ell)$ denotes the number of paths in $\Omega$ with all partial sums at most $\ell$. Let $Q_{p, r}(\ell)$ be the number of paths in $\Omega_{p, r}$ with $S_{j} \geqslant \ell$ for some $j$. Obviously

$$
R_{p, r}(\ell)+Q_{p, r}(\ell+1)=B_{p, r}
$$

\section{Theorem 1.4.}

$$
Q_{p, r}(\ell)= \begin{cases}B_{p, r}, & \text { if } \ell \leqslant \max (r, 0), \\ \sum_{j=\lceil(\ell-r) / t\rceil}^{p} C_{p-j, \ell} B_{j, r-\ell}, & \text { otherwise. }\end{cases}
$$


Proof. The result is clear for $\ell \leqslant \max (r, 0)$ since $S_{0}=0$ and $S_{p t+p+r}=r$. Thus, assume $\ell>\max (r, 0)$. Since $S_{j}$ can increase by ones only, any path that reaches $\ell$ consists of an initial " $+\ell$ " trip. If this initial " $+\ell$ " trip contains $p-j$ " $-t$ " s, then there are $C_{p-j, \ell}$ such initial segments, and each of them is to be followed by a path in $\Omega_{j, r-\ell}$, where $j t+r-\ell \geqslant 0$. Thus, the number of paths that reach $\ell$ equals

$$
\sum_{j \geqslant(\ell-r) / t} C_{p-j, \ell} B_{j, r-\ell}
$$

Let $Q_{p, r}^{=}(\ell)$ be the number of paths in $\Omega_{p, r}$ with $S_{j}=\ell$ for some $j$. Then since $S_{j}$ can increase by ones only, we have

$$
Q_{p, r}^{=}(\ell)=Q_{p, r}(\ell), \quad \ell \geqslant 0 .
$$

Also,

$$
Q_{p, r}^{=}(\ell)=Q_{p, r}(r-\ell), \quad \ell \leqslant r,
$$

as $Q_{p, r}^{=}(\ell)=Q_{p, r}^{=}(r-\ell)$ by the Reversal Lemma. When $r<\ell<0$, the quantity $Q_{p, r}^{=}(\ell)$ is much harder to enumerate, and we shall get back to it later.

Corollary 1.5. If $\ell<\max (r, 0)$, then $R_{p, r}(\ell)=0$. If $\ell \geqslant \max (r, 0)$, then

$$
\begin{aligned}
R_{p, r}(\ell) & =\sum_{0 \leqslant j<\lceil(\ell+1-r) /(t+1)\rceil} C_{p-j, \ell+1} B_{j, r-\ell-1} \\
& =\sum_{0 \leqslant j<\lceil(\ell+1-r) /(t+1)\rceil}(-1)^{j} C_{p-j, \ell+1}\left(\begin{array}{c}
\ell-r-j t \\
j
\end{array}\right) .
\end{aligned}
$$

Proof. The case for $\ell<\max (r, 0)$ is clear. Assume $\ell \geqslant \max (r, 0)$. Then we have

$$
R_{p, r}(\ell)=B_{p, r}-Q_{p, r}(\ell+1) .
$$

Since

$$
\sum_{j=0}^{p} C_{p-j, \ell+1} B_{j, r-\ell-1}=B_{p, r}
$$

it follows from Theorem 1.4, where $\ell$ is replaced by $\ell+1$, that

$$
R_{p, r}(\ell)=\sum_{0 \leqslant j<(\ell+1-r) / t} C_{p-j, \ell+1} B_{j, r-\ell-1}
$$

The summation index $j$ satisfies $j t+r-\ell-1<0$, and for this range of $j$, the coefficient $B_{j, r-\ell-1}$ is nonzero only when $j(t+1)+r-\ell-1<0$. Thus,

$$
R_{p, r}(\ell)=\sum_{0 \leqslant j<\lceil(\ell+1-r) /(t+1)\rceil} C_{p-j, \ell+1} B_{j, r-\ell-1} .
$$

The last equality in the Corollary now follows from

$$
B_{j, r-\ell-1}=\left(\begin{array}{c}
j t+j+r-\ell-1 \\
j
\end{array}\right)=(-1)^{j}\left(\begin{array}{c}
\ell-r-j t \\
j
\end{array}\right) .
$$


Note that if $\max (0, r) \leqslant \ell \leqslant r+t$, then the sums in the Corollary have only one term. Thus,

$$
R_{p, r}(\ell)=C_{p, \ell+1}, \quad \text { provided } \max (0, r) \leqslant \ell \leqslant r+t,
$$

which is independent of $r$. These numbers are related to the solutions to the ballot problem. Recall that in the ballot problem, two candidates $A$ and $B$ square off in an election, with $A$ receiving $a$ votes and $B$ receiving $b$ votes (with $a=p t+r, b=p$ in our notation). The original question was to find the probability that, as the votes are counted, $A$ has more than $t$ times as many votes as $B$ throughout the tally, assuming that $r \geqslant 1$. The question amounts to calculating the number of sequences in $\Omega_{p, r}$ with partial sums $S_{j} \geqslant 1$ for all $j \geqslant 1$. Since these sequences must start with a " +1 ," the number of these sequences equals the number of sequences in $\Omega_{p, r-1}$ with $S_{j} \geqslant 0$, which equals, from Corollaries 1.2 and 1.5,

$$
R_{p, r-1}^{\prime}(0)=R_{p, r-1}(r-1)=C_{p, r}, \quad \text { provided } r \geqslant 1
$$

This is the well-known solution to the ballot problem. If we assume in the spirit of the ballot problem that each vote for $A$ has weight 1 and each vote for $B$ has weight $t$, then the number of ways for which the votes are tallied so that $A$ is ahead of $B$ by a weight of at most $\ell$ at all times equals the number $\hat{R}_{p, r}(\ell)$ of sequences for which $S_{j} \leqslant \ell$, for all

$j \geqslant 1$. If $\ell \geqslant 0$, then $\hat{R}_{p, r}(\ell)=R_{p, r}(\ell)$, and if $\ell<0$, then $\hat{R}_{p, r}(\ell)=R_{p-1, r+t}(\ell+t)$ as the sequences counted must start with a "-t." The relationship between crossings and crossing sums (see later sections) can be related to the ballot problem similarly.

We would like to mention in passing that our results can also be translated to the case where $t$ is the reciprocal of an integer. For $\omega \in \Omega$, define the "dual" sequence $\tilde{\omega}$ so that $\tilde{w}_{i}=1$ if $\omega_{i}=1$, and $\tilde{\omega}_{i}=-1 / t$ if $\omega_{i}=-t$. Then the partial sums $\tilde{S}_{j}$ for $\tilde{\omega}$ satisfies $\tilde{S}_{j} \leqslant \ell$ if and only if $S_{j} \geqslant-\ell t$. Using the dual sequences, one can obtain an explicit solution to the ballot problem when the parameter $t$ is the reciprocal of an integer.

\section{Paths with a Given Number of Crossings}

We say that an upcrossing about $\ell$ occurs at $\nu$ if $S_{\nu} \leqslant \ell$ and $S_{\nu+1}>\ell$. Since $S_{\nu}$ can only increase by ones, the definition of an upcrossing about $\ell$ is the same as $S_{\nu}=\ell$ and $S_{\nu+1}=\ell+1$. Similarly, we say that a downcrossing about $\ell$ occurs at $\nu$ if $S_{\nu} \geqslant \ell$ and $S_{\nu+1}<\ell$. We are also interested in the crossing number associated with a crossing. Since each upcrossing has $S_{\nu}=\ell$ and $S_{\nu+1}=\ell+1$, the upcrossing number is always 1. For a downcrossing about $\ell$ occurring at $\nu$, it is possible that $S_{\nu}=\ell+t-x$ and $S_{\nu+1}=\ell-x$, where $1 \leqslant x \leqslant t$. In this case, we say that the downcrossing is accompanied with a downcrossing number $x$. Since all upcrossing numbers equal 1, we shall be interested only in downcrossing numbers.

For any $\ell, n \geqslant 0$ and $k \geqslant 1$, we write $q=n+k(t+1)$ and let

$$
T=\left\{\omega \in \Omega: S_{n}=S_{q}=\ell, S_{j} \neq \ell \text { for } n<j<q\right\} .
$$


Note that for $\omega \in T$, it is necessary that the subsequence $\omega_{n+1}, \ldots, \omega_{q}$ has exactly $k t$ " +1 "s and $k$ " $-t$ "s. We next partition $T$ into sets $A_{x}$, where $0 \leqslant x \leqslant t$, by defining

$$
A_{x}=\left\{\omega \in T: S_{\nu}=\ell+t-x \text { and } S_{\nu+1}=\ell-x \text { for some } n \leqslant \nu<q\right\} .
$$

We note that there are two cases for each $\omega \in T$. If $\omega_{q}=+1$, then a downcrossing has occurred at $\nu$ for some $\nu<q$, and $A_{x}$ is simply the set of those paths with downcrossing number equal to $x$, where $x=1,2, \ldots, t$. Otherwise, we have $\omega_{q}=-t$, and $\omega$ does not have a downcrossing in the interval $[n, q)$ (as $S_{j}>\ell$ for $n<j<q$ ), and $A_{0}$ is the set of these paths. It is therefore clear that $\left\{A_{x}\right\}_{x=0}^{t}$ is a partition of $T$. The following lemma says that the sets $A_{x}$ are equinumerous, and it is a direct consequence of the Reversal Lemma.

Lemma 2.1 (The Crossing Lemma). Let $T$ and $A_{0}, A_{1}, \ldots, A_{t}$ be as defined above. Then $\left\{A_{x}\right\}_{x=0}^{t}$ is a partition of $T$ and

$$
\left|A_{x}\right|=\frac{1}{t+1}|T|, \quad 0 \leqslant x \leqslant t .
$$

Remark. The result of the Crossing Lemma does not depend on the choice of $\ell, n$, or $k$. The only property required is that $S_{n}=S_{q}$ and $S_{j} \neq S_{n}$ for $n<j<q$. We shall sometimes consider the sets $A_{x}$ of paths as events, meaning the set of of paths with the property specified in the definition of $A_{x}$. For future reference, note also that $A_{0}$ corresponds to the set of strict Dyck paths on $[n, q]$, and $A_{t}$ corresponds to the set of strict reverse Dyck paths on $[n, q]$, and thus $\left|A_{x}\right|=C_{k-1, t}$ for $0 \leqslant x \leqslant t$.

Proof. We have already shown that $\left\{A_{x}\right\}_{x=0}^{t}$ is a partition of $T$. We shall prove the second part of the Lemma by showing that $\left|A_{x}\right|=\left|A_{0}\right|$ for each $x$. We shall assume without loss of generality that $\ell=0$ and $k>0$.

Note first that for $\omega \in T$, we have $\omega \in A_{0}$ if and only if $\omega_{q}=-t$. Given that $\omega \in A_{x} \subseteq T$, where $x \neq 0$, find $\nu$ so that $n \leqslant \nu \leqslant q$ and $S_{\nu}=t-x, S_{\nu+1}=-x$. Now consider the path $\omega(\nu, q]$. We shall first show that $\omega(\nu, q] \in T$. By property of $\omega \in T$, we know that $S_{n}(\omega(\nu, q])=0$ and $S_{j}(\omega(\nu, q]) \neq 0$ for $n<j \leqslant \nu$, and by the Reversal Lemma, we have

$$
S_{j}(\omega(\nu, q])=S_{\nu}(\omega)-S_{\nu+q-j}(\omega), \quad \nu \leqslant j \leqslant q .
$$

The above shows that $S_{q}(\omega(\nu, q])=0$ and $S_{j}(\omega(\nu, q])>0$ for $\nu<j<q$ because $S_{\nu}(\omega)=$ $t-x \geqslant 0$ and $S_{\nu+q-j}(\omega)<0$. Thus, $S_{n}(\omega(\nu, q])=S_{q}(\omega(\nu, q])=0$ and $S_{j}(\omega(\nu, q]) \neq 0$ for $\nu<j<q$. Hence $\omega(\nu, q] \in T$. Since the $q^{\text {th }}$ digit of $\omega(\nu, q]$ is " $-t$," we have also that $\omega(\nu, q] \in A_{0}$. Furthermore, given that $\omega(\nu, q]$ is obtained from $\omega \in A_{x}$, we can find $\nu$ by noting from (8) that $\nu$ is the largest value of $j<q$ for which

$$
S_{j}(\omega(\nu, q])=S_{\nu}(\omega)=t-x .
$$

Therefore, we can invert the map and recover $\omega$ from $\omega(\nu, q]$. Thus, the map is injective. Given $\omega^{\prime}$ in $A_{0}$, since $S_{q-1}\left(\omega^{\prime}\right)=t$ and the partial sums increase by ones, we must have 
$S_{j}\left(\omega^{\prime}\right)=t-x$ at some point between $n$ and $q$. Therefore, the map is surjective as well. Thus, $\left|A_{x}\right|=\left|A_{0}\right|$ for all $x$. The desired conclusion now follows. Figure 1 gives an example of the map from $A_{2}$ to $A_{0}$.

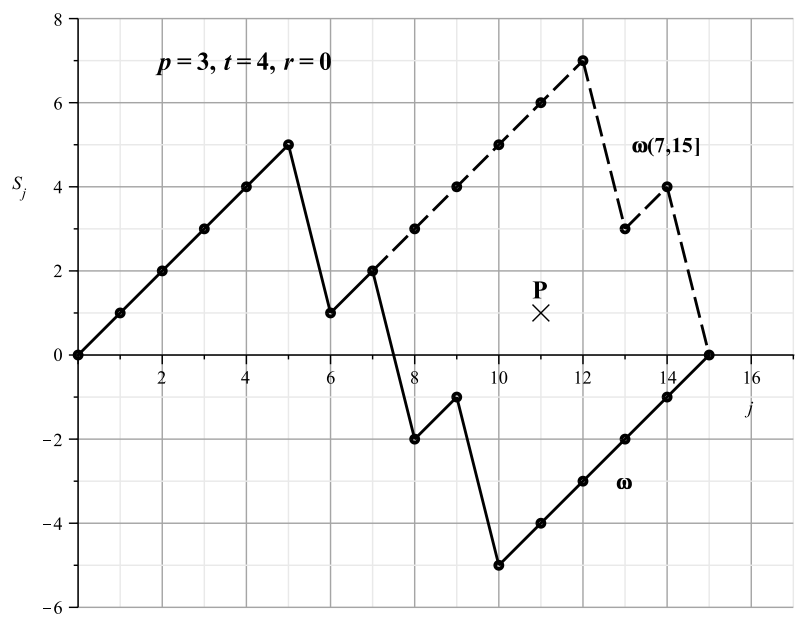

Figure 1: The figure shows a path $\omega \in A_{2}$, with $n=0, q=15$, $\ell=0$ and $\nu=7$. The sequence $\omega(7,15] \in A_{0}$ is obtained after a rotation about (or reflection through) the point $P$ (or after reversing the subsequence $\left.\omega_{8}, \omega_{9}, \ldots, \omega_{15}\right)$.

Alternatively, one can show that there a bijection from $A_{x}$ to $A_{x-1}$, where $1 \leqslant x \leqslant t$, as follows: For a path from $A_{x}$, there is a " $+x$ " trip following the crossing to $-x$. Taking the last " +1 " trip, reversing it, and sending it to the beginning of the path results in a path in $A_{x-1}$, a process that can be reversed.

The Crossing Lemma describes one way the midpoint reflection method is typically implemented. It is a useful tool in counting paths as it allows us to break paths into successive segments $[i, j]$ where $S_{i}=S_{j}=\ell$ and $S_{h} \neq \ell$ for $i<h<j$, and the set of subpaths on each segment can be partitioned into the equinumerous classes $A_{x}, 0 \leqslant x \leqslant t$. The correspondence of paths indicated by the Crossing Lemma has been noted before, dating back to the solution of the ballot problem. Mohanty, in [6, eq. (18)], gave a non-geometric proof of the Crossing Lemma by deleting the downcrossing and using the convolution (4).

When $t \geqslant 1$ is a positive integer, the proof of the ballot theorem follows easily with the Crossing Lemma in place. For any "bad" ballot permutation, that is, a vote count for which $A$ does not always lead, there is a first tie after the ballot count has begun. Using the Crossing Lemma on the section between the start and this first tie, we establish a $(t+1)$-to-one correspondence from the bad ballot permutations to the ballot permutations that start with a vote for $B$. Writing $r=a-b t \geqslant 0$, there are $B_{b-1, r+t}$ of the latter, and we obtain the familiar answer for the number of "good" ballot permutations,

$$
B_{b, r}-(t+1) B_{b-1, r+t}=C_{b, r} .
$$


For any $\ell$, let

$$
\begin{aligned}
n_{\ell}(\omega) & =\left|\left\{j: S_{j}(\omega)=\ell\right\}\right|, \\
n_{\ell}^{+}(\omega) & =\left|\left\{j: S_{j}(\omega)=\ell, S_{j+1}(\omega)=\ell+1\right\}\right|, \\
n_{\ell}^{-}(\omega) & =\left|\left\{j: S_{j}(\omega)=\ell, S_{j+1}(\omega)=\ell-t\right\}\right| .
\end{aligned}
$$

Note that since $S_{p t+p+r}(\omega)=r$ always, we have

$$
n_{\ell}(\omega)=n_{\ell}^{+}(\omega)+n_{\ell}^{-}(\omega)+\delta_{\ell, r} .
$$

Let $N_{\ell}(k), N_{\ell}^{+}(k)$, and $N_{\ell}^{-}(k)$ denote the number of paths $\omega$ with, respectively, $n_{\ell}(\omega)$, $n_{\ell}^{+}(\omega)$, and $n_{\ell}^{-}(\omega)$ equal to $k$. Similarly, let $H_{\ell}(k), H_{\ell}^{+}(k)$ and $H_{\ell}^{-}(k)$ denote the number of paths with $n_{\ell}, n_{\ell}^{+}$, and $n_{\ell}^{-}$at least $k$. Both $N_{\ell}(k)$ and $N_{\ell}^{+}(k)$ were explored in Niederhausen [8, Examples 1 and 2]. These quantities depend on the parameters $p$ and $r$. In situations where there is a need to state these parameters explicitly, we shall write for example $n_{p, r, \ell}, N_{p, r, \ell}(k), H_{p, r, \ell}(k)$, etc.

Theorem 2.2. Suppose that $0 \leqslant \ell \leqslant r$. Then for $k \geqslant 0$,

$$
\begin{aligned}
N_{\ell}(k+1) & =(t+1)^{k} C_{p-k, k t+r}, \\
H_{\ell}(k+1) & =(t+1)^{k} B_{p-k, k t+r}, \\
N_{\ell}^{+}\left(k+1-\delta_{\ell, r}\right) & =t^{k} C_{p-k, k t+k+r+1}, \\
H_{\ell}^{+}\left(k+1-\delta_{\ell, r}\right) & =t^{k} B_{p-k, k t+k+r}, \\
N_{\ell}^{-}(k) & =\sum_{j=k}^{p}\left(\begin{array}{l}
j \\
k
\end{array}\right) t^{j-k} C_{p-j, j t+r}, \\
H_{\ell}^{-}(k) & =\sum_{j=k}^{p}\left(\begin{array}{l}
j-1 \\
k-1
\end{array}\right) t^{j-k} B_{p-j, j t+r} .
\end{aligned}
$$

Proof. We note first that $n_{\ell}^{+} \geqslant 1$ unless $r=\ell$, which is the reason for the term $\delta_{\ell, r}$ appearing (11) and (12). To show (9), we note that for each path counted by $N_{\ell}(k+1)$, there are exactly $k$ segments $[i, j]$ where

$$
S_{i}=\ell, S_{j}=\ell, \text { and } S_{h} \neq \ell, \quad i<h<j .
$$

Let $M$ be the set of paths with the additional condition that for each segment $[i, j], S_{h}<\ell$ for $i<h<j$. That is, in terms of the notation in the Crossing Lemma, the event $A_{t}$ occurs for each of the $k$ segments. We claim that $N_{\ell}(k+1)=(t+1)^{k}|M|$. This is because by applying the Crossing Lemma to each of the $k$ segments, every $\omega \in \Omega$ with $n_{\ell}=k+1$ corresponds to a $\omega \in M$, and each $\omega \in M$ corresponds to $(t+1)^{k}$ paths with $n_{\ell}=k+1$. It therefore remains to show that $|M|=C_{p-k, k t+r}$. 
Suppose $\omega \in M$. Then $\omega$ is comprised of an initial " $+\ell$ " trip, $k$ strict reverse Dyck paths, then a reverse " $+(r-\ell)$ " trip. Then using $(5)$ and Theorem 1.3,

$$
\begin{aligned}
|M| & =\sum_{\substack{q_{i} \geqslant 0, p_{i} \geqslant 1 \\
q_{0}+p_{1} \cdots+p_{k}+q_{1}=p}} C_{q_{0}, \ell} C_{p_{1}-1, t} \cdots C_{p_{k}-1, t} C_{q_{1}, r-\ell} \\
= & \sum_{\substack{p_{i} \geqslant 0 \\
p_{0}+\cdots+p_{k+1}=p-k}} C_{p_{0}, \ell} C_{p_{1}, t} \cdots C_{p_{k}, t} C_{p_{k+1}, r-\ell} \\
= & C_{p-k, k t+r} .
\end{aligned}
$$

The above can also be shown combinatorially. Upon removal of the $k$ " $-t$ "s that cause the event $A_{t}$ to occur in each of the $k$ segments, and reversal of the final reverse " $+(r-\ell)$ " trip, we have a path in $\Omega_{p-k, k t+r}$ that is a " $+(k t+r)$ " trip. Since each path of $\Omega_{p-k, k t+r}$ that is a " $+(k t+r)$ " trip can be decomposed into a " $+\ell$ " trip, followed by $k$ " $+t$ " trips, and a " $+(r-\ell)$ " trip, we can reverse the last trip and insert the missing " $+t$ " $\mathrm{s}$ in the appropriate spots. Thus, we have a bijection. It follows that $|M|=C_{p-k, k t+r}$. Figure 2 below gives an example of an $\omega \in M$.

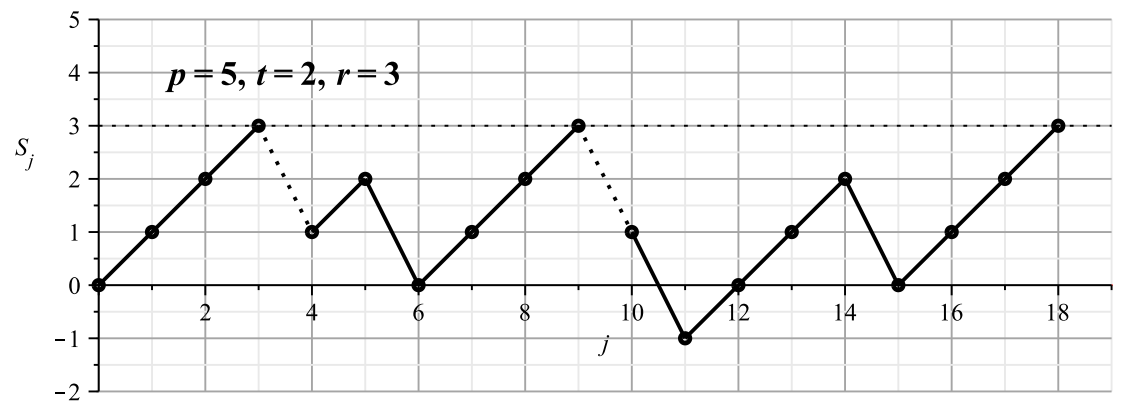

Figure 2: The figure shows an $\omega \in M$ with $\ell=r=3$ and $k=2$. Note that $\omega_{4}=\omega_{10}=-t$ and they cause the event $A_{t}$ to occur twice. The removal of $\omega_{4}$ and $\omega_{10}$ results in a " $+\ell$ " trip and $k$ " $+t$ " trips.

Equation (10) follows from (9) by noting the finite difference

$$
\begin{aligned}
\Delta_{k}\left((t+1)^{k} B_{p-k, k t+r}\right) & =(t+1)^{k+1} B_{p-k-1,(k+1) t+r}-(t+1)^{k} B_{p-k, k t+r} \\
& =-(t+1)^{k} C_{p-k, k t+r}
\end{aligned}
$$

and that we have $H_{\ell}(1)=B_{p, r}$. We can also prove (10) directly as follows. We first use the Crossing Lemma to show that $H_{\ell}(k+1)=(t+1)^{k}\left|M^{\prime}\right|$ where $M^{\prime}$ is the set of paths composed of an initial " $+\ell$ " trip, $k$ strict reverse Dyck paths, and finally a path from $\ell$ to $r$. The result is then proved by showing $\left|M^{\prime}\right|=B_{p-k, k t+r}$. We omit the details. 
To prove (11), we shall count the set $M^{+}$of paths in $\Omega_{p, r}$ that consist of a " $+\ell$ " trip, a reverse Dyck path, $k$ segments where each segment consists of a strict Dyck path followed by a reverse Dyck path, then a reverse " $+(r-\ell)$ " trip. We have

$$
\begin{aligned}
\left|M^{+}\right| & =\sum_{\substack{p_{0}+\cdots+p_{k+1} \\
+q_{0}+\cdots+q_{k}=p}} C_{p_{0}, \ell} C_{q_{0}, 1} C_{p_{1}-1, t} C_{q_{1}, 1} \cdots C_{p_{k}-1, t} C_{q_{k}, 1} C_{p_{k+1}, r-\ell} \\
& =C_{p-k, k t+k+r+1},
\end{aligned}
$$

where the sum is over $p_{0} \geqslant 0, p_{k+1} \geqslant 0, p_{i} \geqslant 1$ for $1 \leqslant i \leqslant k$ and $q_{i} \geqslant 0$ for $0 \leqslant i \leqslant k$, and the last equality follows from (5). Equation (11) now follows from $N_{\ell}^{+}=t^{k} \mid M^{+}$. Indeed, for $\omega \in M^{+}$, each strict Dyck path corresponds to the event $A_{0}$ in the Crossing Lemma and contributes one toward $n_{\ell}^{+}$. The events $A_{0}, A_{1}, \ldots, A_{t-1}$ (but not $A_{t}$ ) each can contribute 1 to $n_{\ell}^{+}$. It therefore follows from the Crossing Lemma that in counting $N_{\ell}^{+}$, each strict Dyck path in $\omega \in M^{+}$contributes a factor of $t$, thus giving $N_{\ell}^{+}=t^{k}\left|M^{+}\right|$.

Equation (12) follows from (11), the finite difference

$$
\Delta_{k}\left(t^{k} B_{p-k, k t+k+r}\right)=-t^{k} C_{p-k, k t+k+r+1}
$$

and that $H_{\ell}^{+}\left(1-\delta_{\ell, r}\right)=B_{p, r}$. This result can also be obtained directly.

To prove (13), we note that in our proof of (9), if the paths in the set $M$ each contain $j$ (instead of $k$ ) segments, then $|M|=C_{p-j, j t+r}$. For each $\omega \in M$, there are $\left(\begin{array}{l}j \\ k\end{array}\right)$ ways to fix $k$ segments for which the event $A_{t}$ occurs, and the other $j-k$ segments each can have $t$ possible choices: $A_{0}, A_{1}, \ldots, A_{t-1}$. The Crossing Lemma gives that the events $A_{i}$ are equally likely. This means that each $\omega \in M$ contributes $\left(\begin{array}{l}j \\ k\end{array}\right) t^{j-k}$ paths counted by $N_{\ell}^{-}(k)$. Summing $\left(\begin{array}{l}j \\ k\end{array}\right) t^{j-k} C_{p-j, j t+r}$ over $j$ thus gives the result.

The proof of (14) is similar to that of (13). For $j \geqslant k$, let $M^{\prime}$ be the set of paths in $\Omega_{p, r}$ where each path is composed of an initial " $+\ell$ " trip, $j$ strict reverse Dyck paths, followed by a path from $\ell$ to $r$. The paths in $M^{\prime}$ satisfy $n_{\ell} \geqslant j+1$. As commented at our proof of (10), we have $\left|M^{\prime}\right|=B_{p-j, j t+r}$. Each strict reverse Dyck path produces a downcrossing number of $t$. Thus, out of the $j$ strict reverse Dyck paths, $k$ of them will remain, and in order to avoid double counting, we need to insist that the last of the $j$ strict reverse Dyck paths to remain as it is. This accounts for the factor $\left(\begin{array}{l}j-1 \\ k-1\end{array}\right)$. For each of the remaining $j-k$ strict reverse Dyck paths, we need to switch it to a path associated with one of the events $A_{0}, A_{1}, \ldots, A_{t-1}$ defined in the Crossing Lemma, thus accounting for the factor $t^{j-k}$. We complete the proof by summing $\left(\begin{array}{l}j-1 \\ k-1\end{array}\right) t^{j-k} B_{p-j, j t+r}$ over $j$.

The result for $N_{\ell}(k)$ when $0 \leqslant \ell \leqslant r$ is [6, Theorem 3], while our counting of $M$ is [6, Corollary 1 (ii)].

It is possible to obtain formulas for $N^{+}, H^{+}, N^{-}, H^{-}$through other means. In this respect, the formulas in the following theorem represent some combinatorial identities in association with Theorem 2.2. 
Theorem 2.3. Suppose that $0 \leqslant \ell \leqslant r$. Then for $k \geqslant 0$,

$$
\begin{aligned}
& N_{\ell}^{+}\left(k+1-\delta_{\ell, r}\right)=t^{k} \sum_{j=k}^{p}\left(\begin{array}{l}
j \\
k
\end{array}\right) C_{p-j, j t+r}, \\
& H_{\ell}^{+}\left(k+1-\delta_{\ell, r}\right)=t^{k} \sum_{j=k}^{p}\left(\begin{array}{l}
j-1 \\
k-1
\end{array}\right) B_{p-j, j t+r} .
\end{aligned}
$$

Suppose that $0 \leqslant \ell<r+t$. Then for $k \geqslant 0$,

$$
\begin{aligned}
& N_{\ell}^{-}(k)=\sum_{j=k}^{p}\left(\begin{array}{l}
j \\
k
\end{array}\right)(t-1)^{j-k} C_{p-j, j t+j+r+1}, \\
& H_{\ell}^{-}(k)=\sum_{j=k}^{p}\left(\begin{array}{l}
j-1 \\
k-1
\end{array}\right)(t-1)^{j-k} B_{p-j, j t+j+r} .
\end{aligned}
$$

Proof. Equations (15) and (16) are proved in the same way as (13) and (14) are proved.

To prove (17), we note that a downcrossing about $\ell$ with a downcrossing number $t$ is the same as a downcrossing about $\ell^{\prime}=\ell-t+x$ with a downcrossing number $x$, when $1 \leqslant x \leqslant t$. We shall show (17) for the case when $r \geqslant 1, t \geqslant 2$ and $\ell=r+t-1$. Here we choose $x=1$, and the number of paths counted by $N_{\ell}^{-}(k)$ equals the number of paths for which there are exactly $k$ downcrossings about $\ell^{\prime}=r$ with downcrossing number 1 . Each of these downcrossings about $\ell^{\prime}$ is associated with a preceding upcrossing about $\ell^{\prime}$. If the paths counted have a total of $j$ upcrossings about $\ell^{\prime}$, then the remaining $j-k$ upcrossings each has $t-1$ choices, as the events $A_{1}$ and $A_{t}$ defined in the Crossing Lemma are ruled out. Therefore, using the method in counting $M^{+}$as in the proof of (11) (where paths in $M^{+}$have $j$ segments), we see that number of paths with $j$ upcrossings about $\ell^{\prime}, k$ of which have downcrossing number 1 , equals $\left(\begin{array}{l}j \\ k\end{array}\right)(t-1)^{j-k} C_{p-j, j t+j+r+1}$. Equation (17) now follows by summing over $j$. The above argument shows that the right hand sides of (13) and (17) are equal for infinitely many values of $r$ and $t$, and we use a polynomial argument to complete the proof.

The proof of equation (18) is similar to that of (14) using the idea of the proof of (17). We omit the details.

Remark. The idea of using the sets $M$ and $M^{+}$(multiple times in some instances) as "templates" enabled us to prove (13), (14), and Theorem 2.3. We can use this same procedure to count other objects. For example, let $N_{\ell}(k, m)$ denote the number of paths in $\Omega$ with $n_{\ell}^{+}=k$ and $n_{\ell}^{-}=m$. Then, for $0 \leqslant \ell \leqslant r$, we have

$$
N_{\ell}\left(k+1-\delta_{\ell, r}, m\right)=t^{k}\left(\begin{array}{c}
k+m \\
m
\end{array}\right) C_{p-k-m,(k+m) t+r},
$$

so that summing over $m$ gives (15), and summing over $k$ gives (13). Equation (19) may be shown by starting with the set $M$ used in the proof of (9), with $k$ replaced by $k+m$. This set $M$ has cardinality $C_{p-k-m,(k+m) t+r}$. With $k+m$ segments, we select $m$ of them 
to be of the type $A_{t}$, while for the remaining $k$ segments, we have $t$ choices of the events $A_{0}$ through $A_{t-1}$. This generates the factor $t^{k}\left(\begin{array}{c}k+m \\ m\end{array}\right)$. The extra step from $\ell$ to $\ell+1$ is automatic, unless $\ell=r$, thus the involvement of the term $\delta_{\ell, r}$.

When the condition $0 \leqslant \ell \leqslant r$ is not satisfied, we shall concentrate on $H$ and $H^{+}$. Note that $N(k)=H(k)-H(k+1)$ and $N^{+}(k)=H^{+}(k)-H^{+}(k+1)$ and that $N^{-}(k)$ and $H^{-}(k)$ can be calculated from $N(k)$ and $H(k)$ respectively, in the manner of the proofs of (13) and (14). However, since the formulas obtained are rather complicated, we shall not state them explicitly.

Theorem 2.4. Assume that $\ell \geqslant \max (r, 0)$. Then for $k \geqslant 0$,

$$
\begin{aligned}
H_{\ell}(k+1) & =(t+1)^{k} Q_{p-k, k t+r}(k t+\ell) \\
& =(t+1)^{k} \sum_{j=\lceil(\ell-r) / t\rceil}^{p-k} C_{p-k-j, k t+\ell} B_{j, r-\ell}, \\
H_{\ell}^{+}(k+1) & =t^{k} Q_{p-k, k t+k+r}(k t+k+\ell+1) \\
& =t^{k} \sum_{j=\lceil(\ell-r+1) / t\rceil}^{p-k} C_{p-k-j, k(t+1)+\ell+1} B_{j, r-\ell-1} .
\end{aligned}
$$

Remark. The results of Theorem 2.4 hold for any $\ell \geqslant 0$, as when $0 \leqslant \ell<r$ equations (20) and (21) reduce to (10) and (12), respectively.

Proof. Recall that $Q_{p, r}(\ell)$ is the number of paths in $\Omega_{p, r}$ for which $S_{j} \geqslant \ell$ for some $j$, and its formula is given in Theorem 1.4. To prove (20), let $M$ be the set of paths in $\Omega_{p, r}$ for which $N_{\ell} \geqslant k+1$ and after the first $k$ times the partial sum equals $\ell$, a " $-t$ " follows. (That is, the subpath over each of the $k$ intervals $[i, j]$ where $S_{i}=S_{j}=\ell$ is in $A_{t}$.) Then, $H_{\ell}(k+1)$ equals $(t+1)^{k}|M|$ by the Crossing Lemma. A path $\omega \in M$ consists of a " $+\ell$ " trip, $k$ strict reverse Dyck paths, followed by a path from $\ell$ to $r$. Removal of the $k$ " $-t$ "s found at the beginning of each strict reverse Dyck path gives a path from $\Omega_{p-k, k t+r}$ that reaches $k t+\ell$. As this process can be reversed by adding back the " $-t$ "s at the appropriate places, we must have $|M|=Q_{p-k, k t+r}(k t+\ell)$.

To prove (21), we shall count the set $M^{+}$of paths $\omega$ for which $N_{\ell}^{+}(\omega) \geqslant k+1$, with the additional property that the event $A_{0}$ occurs after each of the first $k$ upcrossings. Then $\omega \in M^{+}$consists of a " $+\ell$ " trip, $k$ segments where each segment consists of a reverse Dyck path followed by a strict Dyck path, a " +1 " trip, then finally a path from $\ell+1$ to $r$. For the strict Dyck path in each of the $k$ segments, we remove the "- $t$ " necessarily at the end, then reverse it (thus turning it into a " $+t$ " trip), while for each reverse Dyck path, we attach a " +1 " to the end (thus turning it into a " $+1 "$ trip). This results in a path composed of a " $+(k t+k+\ell+1)$ " trip, followed a path from $\ell+1$ to $r$. That is, the resulting path is from $\Omega_{p-k, k t+k+r}$ and it reaches $k t+k+\ell+1$. As this manipulation can be reversed by adding/removing " $-t$ " s and " +1 "s at the appropriate places, we have

$$
\left|M^{+}\right|=Q_{p-k, k t+k+r}(k t+k+\ell+1)
$$


The Crossing Lemma now gives that

$$
H_{\ell}^{+}(k+1)=t^{k}\left|M^{+}\right|=t^{k} Q_{p-k, k t+k+r}(k t+k+\ell+1),
$$

as desired.

Lemma 2.5. For any $\ell$ and for $k \geqslant 0$,

$$
H_{\ell}(k+1)=H_{r-\ell}(k+1),
$$

and

$$
H_{\ell}^{+}(k+1)=H_{r-\ell-1}^{+}(k+1) .
$$

Proof. This a direct consequence of the Reversal Lemma. Note that for each $\omega \in \Omega$, if we reverse all of $\omega$, we have $S_{j}(\omega)=\ell$ if and only if $S_{p t+p+r-j}\left(\omega^{R}\right)=r-\ell$, from which (22) follows. In addition, we have $S_{j}(\omega)=\ell$ and $S_{j+1}(\omega)=\ell+1$ if and only if $S_{p t+p+r-j}\left(\omega^{R}\right)=r-\ell$ and $S_{p t+p+r-j-1}\left(\omega^{R}\right)=r-\ell-1$, which is an upcrossing about $r-\ell-1$. This shows (23).

Corollary 2.6. For $\ell \leqslant \min (r, 0)$ and $k \geqslant 0$,

$$
\begin{aligned}
H_{\ell}(k+1) & =(t+1)^{k} Q_{p-k, k t+r}(k t+r-\ell) \\
& =(t+1)^{k} \sum_{j=\lceil-\ell / t\rceil}^{p-k} C_{p-k-j, k t+r-\ell} B_{j, \ell} .
\end{aligned}
$$

For $\ell<\min (r, 0)$ and $k \geqslant 0$,

$$
\begin{aligned}
H_{\ell}^{+}(k+1) & =t^{k} Q_{p-k, k t+k+r}(k t+k+r-\ell) \\
& =t^{k} \sum_{j=\lceil-\ell / t\rceil}^{p-k} C_{p-k-j, k(t+1)+r-\ell} B_{j, \ell} .
\end{aligned}
$$

Proof. The Corollary is a direct result of Theorem 2.4 and Lemma 2.5.

To cover all ranges of $\ell$ and $r$, we shall find formulas for $H$ and $H^{+}$when $r \leqslant \ell<0$, for which we need to work with $Q_{p, r}^{=}(\ell)$.

Lemma 2.7. For integer $r \leqslant \ell<0$ we have

$$
Q_{p, r}^{=}(\ell)=\sum_{j=\lceil-\ell / t\rceil}^{p+\lfloor(r-\ell) / t\rfloor}\left(B_{j, \ell}-H_{j, \ell, 0}(2)\right) B_{p-j, r-\ell} .
$$

Proof. Each path counted by $Q_{p, r}^{=}(\ell)$ consists of an initial subpath $\hat{\omega}$ that first arrives at $\ell$ (that is, it does not touch $\ell$ until the end), followed by a path from $\ell$ to $r$. If $\hat{\omega}$ contains $j$ " $-t$ "s, then it is a path in $\Omega_{j, \ell}$ with $n_{j, \ell, \ell}(\hat{\omega})=1$. Since $n_{j, \ell, \ell}(\hat{\omega})=n_{j, \ell, 0}\left(\hat{\omega}^{R}\right)$, there are $B_{j, \ell}-H_{j, \ell, 0}(2)$ possible choices for $\hat{\omega}$. The result now follows by summing over the appropriate values of $j$. 
Theorem 2.8. For integers $r \leqslant \ell<0$ and $k \geqslant 0$,

$$
\begin{aligned}
& H_{\ell}(k+1)=(t+1)^{k} \sum_{j=k}^{p+\lfloor r / t\rfloor} C_{j-k, k t} Q_{p-j, r}^{=}(\ell), \\
& H_{\ell}^{+}(k+1)=t^{k} \sum_{j=k}^{p+\lfloor(r-1) / t\rfloor} C_{j-k, k(t+1)+1} Q_{p-j, r-1}^{=}(\ell) .
\end{aligned}
$$

Proof. For the paths counted by $H_{\ell}(k+1)$, the first $k+1$ times when the partial sum equals $\ell$ form $k$ segments $[a, b]$ where $S_{a}=S_{b}=\ell$. As before, we count the set $M$ of paths with the restriction that each of the $k$ segments is a strict reverse Dyck path, and then (24) follows from the Crossing Lemma. To count $M$, if there are $j$, where $j \geqslant k$, "- $t$ "s in these $k$ segments, then the removal of these $k$ segments from a path in $M$ results in a path $\omega \in \Omega_{p-j, r}$ passing through $\ell$, and there are $Q_{p-j, r}^{=}(\ell)$ such $\omega$ 's. To count the number of ways to form the $k$ segments, we note that removal of the " $-t$ " at the front of each of the $k$ strict reverse Dyck paths results in a path in $\Omega_{j-k, k t}$ that is itself a " $+k t$ " trip, and that this process can be reversed by inserting the " $-t$ " $s$ at the appropriate places. It follows that there are $C_{j-k, k t}$ ways to form the $k$ segments. We obtain $|M|$ by summing $C_{j-k, k t} Q_{p-j, r}^{=}(\ell)$ over $j$.

For (25), we count the set $M^{+}$of paths $\omega$ for which the event $A_{0}$ follows each of the first $k$ times that $\omega$ passes from $\ell$ to $\ell+1$. The Crossing Lemma then gives (25). Each $\omega \in M^{+}$is composed of an initial trip that first reaches $\ell, k$ segments where each segment consists of a reverse Dyck path followed by a strict Dyck path, a " +1 " trip, and finally a path from $\ell+1$ to $r$. If there are $j$ " $-t$ "s in the $k$ segments together with the " +1 " trip that follows, then removing these components from $\omega$ results in a path $\omega^{\prime} \in \Omega_{p-j, r-1}$ that takes the partial sum $\ell$, and there are $Q_{p-j, r-1}^{=}(\ell)$ such $\omega^{\prime}$. To find the number of ways to form the $k$ segments together with the " +1 " trip that follows, we remove the final " $t$ " from each of the $k$ strict Dyck paths and then reverse the path that remains, thus forming $k$ " $+t$ " trips. Add " +1 " to each of the reverse Dyck path in the $k$ segments, forming $k$ " +1 " trips. This manipulation turns the " $k$ segments with the " +1 " trip that follows' into a path in $\Omega_{j-k, k t+k+1}$ that is itself a " $+(k t+k+1)$ " trip. As this process can be reversed, the number of ways to form the $k$ segments together with the "+1" trip that follows equals $C_{j-k, k t+k+1}$. We then obtain $\left|M^{+}\right|$by summing $C_{j-k, k t+k+1} Q_{p-j, r-1}^{=}(\ell)$ over $j$.

\section{Crossing Sums}

We investigate in this section the sum of the crossing numbers about $\ell$. For $\omega \in \Omega$, we define the upcrossing sum of $\omega$ about $\ell$ as the sum of all upcrossing numbers of $\omega$ about $\ell$, and similarly, the downcrossing sum of $\omega$ about $\ell$ as the sum of all downcrossing numbers of $\omega$ about $\ell$. Since all upcrossing numbers equal 1, the upcrossing sum of $\omega$ is simply $n_{\ell}^{+}(\omega)$ and $N_{\ell}^{+}(k)$ gives the number of paths $\omega$ with upcrossing sum equal to $k$. We shall therefore concentrate on downcrossing sums, and all crossing sums for the rest of the 
section refer to downcrossing sums. In particular, the crossing sums about $r$ are related to the distribution of the gain for the acceptance urn model with " +1 " and " $t$ " balls, using an optimal strategy to maximize the expected gain.

For $\omega \in \Omega_{p, r}$, we call $K_{\ell}(\omega)=\left(x_{1}, \ldots, x_{h}\right)$ the crossing sequence of $\omega$ about $\ell$ if $\omega$ has $h$ downcrossings about $\ell$, and $x_{1}, \ldots, x_{h}$ is the sequence of the $h$ downcrossing numbers. The crossing sum of $\omega$ about $\ell$ is

$$
g_{\ell}(\omega)=\sum_{i=1}^{h} x_{i}, \quad \text { where } K_{\ell}(\omega)=\left(x_{1}, \ldots, x_{h}\right) .
$$

When $t=1$, we can calculate the crossing sums with the help of the reflection method. For an example, see [12, Lemma 2.2]. For integer $t \geqslant 1$, we can calculate crossing sums with the help of rotation.

Theorem 3.1. If $0 \leqslant \ell \leqslant r$, then the number of paths in $\Omega_{p, r}$ with $g_{\ell}(\omega)=k$ equals $Q_{p, r}(r+k)-Q_{p, r}(r+k+1)$. That is, there is a one-to-one correspondence between paths with a crossing sum about $\ell$ of $k$ and paths with a maximum partial sum of $r+k$.

Proof. It suffices to show the one-to-one correspondence indicated by the last statement of the Theorem. We begin by proving the case with $\ell=r$. Suppose $g_{r}(\omega)=k$. We will split $\omega$ into the two subpaths $\omega^{+}$and $\omega^{-}$. For each $i$, we place $\omega_{i}$ into $\omega^{-}$if $S_{i-1}(\omega) \geqslant r$, and we place $\omega_{i}$ into $\omega^{+}$otherwise. All of this is done with the relative order preserved, that is, $\omega^{+}=\omega_{b_{1}} \cdots \omega_{b_{j}}$ is such so that $b_{1}<\cdots<b_{j}$, and similarly for $\omega^{-}$. We then form the path $\bar{\omega}=\omega^{+}\left(\omega^{-}\right)^{R}$.

Suppose $K_{r}(\omega)=\left(x_{1}, \ldots, x_{h}\right)$. If $r \neq 0$, then $\omega^{+}$begins with the initial " $+r$ " trip. The remaining portion of $\omega^{+}$consists of a " $+x_{1}, "$ " $+x_{2}, " \ldots$, and " $+x_{h}$ " trips in $\omega$ following each downcrossing about $r$. Therefore, $\omega^{+}$is a " $+(r+k)$ " trip since $g_{r}(\omega)=k$. For each " $+x_{i}$ " trip in $\omega^{+}$, there is a corresponding " $-x_{i}$ " path in $\omega^{-}$for which only the last partial sum is negative. These " $-x_{1}, " \ldots$, " $-x_{h}$ " paths, followed by a Dyck path, comprise $\omega^{-}$. Due to the properties of these " $-x_{i}$ " paths, all partial sums over $\omega^{-}$are at least $-k$, and in particular the sum over all of $\omega^{-}$equals $-k$. Therefore, $\left(\omega^{-}\right)^{R}$ has the property that all partial sums are at most zero, by the Reversal Lemma. Thus, upon concatenation, $\bar{\omega}$ has a maximum partial sum of $r+k$. Figure 3 gives an example of how $\omega$ is split into $\omega^{+}$ and $\omega^{-}$.

We next show that the map is surjective. Let $\bar{\omega}$ be given, with $\max _{n} S_{n}(\bar{\omega})=r+k$. We find find the smallest such $n$ for which $S_{n}(\bar{\omega})=r+k$, and split $\bar{\omega}$ into $\omega^{+}$(before) and $\left(\omega^{-}\right)^{R}$ (after), reversing the latter to produce $\omega^{-}$. We construct $\omega$ with $g_{r}(\omega)=k$ as follows: We fill in $\omega$ using $\omega^{+}$until a partial sum of $r$ is reached, then using $\omega^{-}$ until a partial sum less than $r$ is reached. We repeat this process until both $\omega^{+}$and $\omega^{-}$ are empty. When finished, the resulting (and uniquely determined) $\omega$ has the property that $g_{r}(\omega)=k$, since the fills from $\omega^{+}$after the initial " $+r$ " trip equal the sum of the downcrossings.

When $0 \leqslant \ell \leqslant r$, any path $\omega$ begins with a " $+\ell$ " trip, and ends with a reverse " $+(r-\ell)$ " trip, with neither contributing to the crossing sum about $\ell$. Reversing, then moving the 


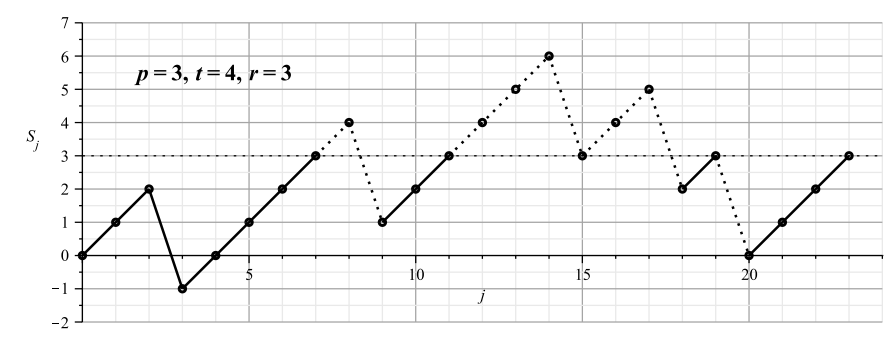

Figure 3: In this example, with $\ell=r=3$ and $k=g_{r}(\omega)=6$, the parts of $\omega$ that are in solid lines are grouped to form $\omega^{+}$and the parts that are in dotted lines are grouped to form $\omega^{-}$. Note that $\bar{\omega}=\omega^{+}\left(\omega^{-}\right)^{R}$ has maximum partial sum equal to $r+k=9$.

now- " $+(r-\ell)$ " trip to the beginning gives a one-to-one correspondence between paths with $g_{\ell}=k$ and those with $g_{r}=k$. This completes the proof.

Theorem 3.2. If $\ell \leqslant \min (r, 0)$ and $k>0$, then the number of paths with $g_{\ell}=k$ equals $Q_{p, r}(r-\ell+k)-Q_{p, r}(r-\ell+k+1)$.

Remark. If $g_{\ell}=0$, then all partial sums must be at least $\ell$, and therefore the number of paths with this property equals $R_{p, r}(r-\ell)$.

Proof. We proceed similarly as in Theorem 3.1, adding $\omega_{n}$ to $\omega^{+}$if and only if $S_{n-1} \geqslant \ell$. This time, $\omega^{+}$will be a " $+k$ " trip, while $\omega^{-}$will have a minimum partial sum of $\ell-k$, ending with a Dyck path followed by a reverse " $+(r-\ell)$ " trip. Reversing the reverse " $+(r-\ell) "$ trip and moving it to the end of $\omega^{+}$gives the two subpaths $\hat{\omega}^{+}, \hat{\omega}^{-}$, and the path $\bar{\omega}=\hat{\omega}^{+}\left(\hat{\omega}^{-}\right)^{R}$ has a maximum partial sum of $r-\ell+k$. Since we can pick off the moved " $+(r-\ell)$ " trip, the map is reversible.

Theorem 3.3. If $r \geqslant 0$ and $k>0$, then the number of paths with $g_{r+1}=k$ equals $Q_{p, r}(r+k)-Q_{p, r}(r+k+1)$. If $r<0$, then the number of paths with $g_{r+1}=k$ equals $Q_{p, r}(k-1)-Q_{p, r}(k)$.

Remark. The number of paths with $g_{r+1}=0$ equals $C_{p, r+1}$ if $r \geqslant 0$, and equals zero when $r<0$.

Proof. Once again, we break $\omega$ into $\omega^{+}$and $\omega^{-}$, placing $\omega_{i}$ into $\omega^{-}$if and only if $S_{i-1}(\omega) \geqslant$ $r+1$. Suppose $r \geqslant 0$. If $K_{r+1}(\omega)=\left(x_{1}, \ldots, x_{h}\right)$ with $g_{r+1}(\omega)=k>0$, then $\omega^{+}$consists of " $+(r+1), "$ " $+x_{1}, " \ldots$, and " $+x_{h-1}$ " trips, followed this time by a " $+\left(x_{h}-1\right)$ " trip, since we return back to $r$ (but not $r+1$ ) after the last downcrossing. Thus, $\omega^{+}$is a " $+(r+k)$ " trip. It now follows similarly to the proofs of Theorems 3.1 and 3.2 that the maximum partial sum of $\bar{\omega}=\omega^{+}\left(\omega^{-}\right)^{R}$ equals $r+k$. If $r<0$, then the initial part of $\omega$ through the first downcrossing about $r+1$ belongs to $\omega^{-}$. Thus $\omega^{+}$will consist only of the " $+x_{1}$," $\ldots$, , $+x_{h-1}$," and " $+\left(x_{h}-1\right)$ " trips, and thus is a " $+(k-1)$ " trip. Therefore, $\bar{\omega}$ will have a maximum partial sum of $k-1$. 
The computation of the crossing sum is more complicated when $\ell>r+1$. Note that in the proofs of the theorems in this section, we do not calculate the downcrossing sum about $\ell$ directly, but rather, we count the return trips back to $\ell$ instead. When $\ell \leqslant r$, there is always a return to $\ell$ following each downcrossing. When $\ell>r$, this may not be the case for the final downcrossing. However, when $\ell=r+1$, we have a guaranteed return to $\ell-1$ (not to $\ell$ ), following the last downcrossing, so we can still calculate the downcrossing sum in that case. Otherwise, when $\ell>r+1$, we do not have any such guarantee. Thus, there is an extra degree of difficulty in obtaining the distribution for the crossing number about $\ell$. Nevertheless, it is still possible to find the number of paths with $g_{\ell}(\omega)=k$. However, such results are not particularly illuminating or of much aesthetic value, thus we omit them from this paper.

\section{The First Downcrossing Number}

We are interested in the first downcrossing number about $\ell$. For integers $\ell$ and $x$ with $1 \leqslant x \leqslant t$, let $X_{p, r}(\ell, x)$ be the number of paths whose first downcrossing number about $\ell$ is $x$. That is, $X_{p, r}(\ell, x)$ is the number of paths for which $\nu=\min \left\{j: S_{j} \geqslant \ell, S_{j+1}<\ell\right\}$ exists and $S_{\nu+1}=\ell-x$. This problem has been studied by Goulden and Serrano [3] when $r \geqslant 0$ and $\ell=0$.

Theorem 4.1. Assume $1 \leqslant x \leqslant t$. Then for $\ell \geqslant 0$,

$$
X_{p, r}(\ell, x)=\sum_{j=0}^{p-1+\min (0,\lfloor(r-\ell+x) / t\rfloor)} C_{j, \ell+t-x+1} B_{p-j-1, r-\ell+x} .
$$

For $\ell \leqslant 0$,

$$
X_{p, r}(\ell, x)=\sum_{j=\max (0,\lceil(\ell-x-r) / t\rceil)}^{p-1+\min (0,\lfloor(\ell+t-x) / t\rfloor)} R_{p-1-j, \ell+t-x}(t-x) B_{j, r-\ell+x} .
$$

Proof. Suppose $\ell \geqslant 0$. Let $1 \leqslant x \leqslant t$ be given. Suppose that $j+1$ " $-t$ "s have been used up to and including the first downcrossing about $\ell$. Any path with a first downcrossing number about $\ell$ of $x$ must begin with a " $\ell$ " trip. A Dyck path, a reverse " $+(t-x)$ " trip, and a " $-t$ " follows, with the last " $-t$ " resulting in the first downcrossing about $\ell$, to $\ell-x$. The total number of paths in $\Omega_{j+1, \ell-x}$ with the above properties equals

$$
\sum_{j_{1}+j_{2}+j_{3}=j} C_{j_{1}, \ell} C_{j_{2}, 1} C_{j_{3}, t-x}=C_{j, \ell+t-x+1}
$$

The portion of $\omega$ following this first downcrossing is a path in $\Omega_{p-j-1, r-\ell+x}$. We thus complete the proof by taking the convolution over the appropriate values of $j$. Figure 4 gives an example of the different parts of $\omega$ used in this proof.

Now we assume $\ell \leqslant 0$. Suppose that there are $j+1$ " $-t$ "s remaining immediately before $\ell$ is crossed. Then a path counted by $X_{p, r}(\ell, x)$ consists of a path in $\Omega_{p-1-j, \ell+t-x}$ 


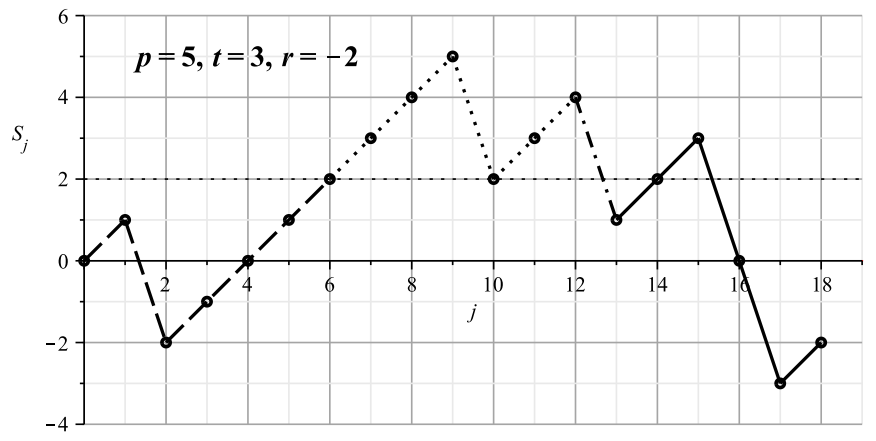

Figure 4: The first downcrossing about $\ell=2$ in this example is caused by $\omega_{13}=-t=-3$, with the first downcrossing number $x=1$ (shown in dashdotted line). The initial " $+\ell$ " trip is depicted in dashed lines, and the Dyck path followed by the reverse "+2" trip are shown in dotted lines.

with partial sums at least $\ell$, (where $j$ must satisfy $j \leqslant p-1+(\ell+t-x) / t$,) followed by a " $-t$ " and then a path in $\Omega_{j, r-\ell+x}$, where $j \geqslant(\ell-r-x) / t$. By the Reversal Lemma, the number of paths in $\Omega_{p-1-j, \ell+t-x}$ with partial sums at least $\ell$ equals the number of paths in $\Omega_{p-1-j, \ell+t-x}$ with partial sums at most $t-x$, which is $R_{p-1-j, \ell+t-x}(t-x)$. As $\left|\Omega_{j, r-\ell+x}\right|=B_{j, r-\ell+x}$, the result follows from summing the product of these two numbers over appropriate values of $j$.

The formulas in Theorem 4.1 have closed forms in some cases. For example, if $\ell \geqslant 0$ and $r \geqslant \ell-x$, we have from (26) that

$$
X_{p, r}(\ell, x)=\sum_{j=0}^{p-1} C_{j, \ell+t-x+1} B_{p-j-1, r-\ell+x}=B_{p-1, r+t+1} .
$$

This extends the result of [3, Theorem 4]. In another example, if $0 \geqslant \ell \geqslant-t+x$ and $r \geqslant \ell-x$, then using (7) and (27),

$$
X_{p, r}(\ell, x)=\sum_{j=0}^{p-1} C_{p-1-j, t-x+1} B_{j, r-\ell+x}=B_{p-1, r-\ell+t+1} .
$$

In another example, suppose that $r<0$. Then all paths cross $r+1$ and each path has a first downcrossing about $r+1$. Then for $1 \leqslant x \leqslant t$

$$
X_{p, r}(r+1, x)=\sum_{j=0}^{p-1+\lfloor(r+1+t-x) / t\rfloor} R_{p-1-j, r+1+t-x}(t-x) B_{j,-1+x} .
$$

If $-t-1 \leqslant r \leqslant-1$, then $(7)$ gives $R_{p-1-j, r+1+t-x}(t-x)=C_{p-1-j, t-x+1}$, and the above becomes

$$
X_{p, r}(r+1, x)=\sum_{j=0}^{p-1+\lfloor(r+1+t-x) / t\rfloor} C_{p-1-j, t-x+1} B_{j,-1+x} .
$$


It follows that for $-t-1 \leqslant r \leqslant-1$,

$$
X_{p, r}(r+1, x)=\left\{\begin{array}{cl}
\left(\begin{array}{c}
p t+p-1 \\
p-1
\end{array}\right), & \text { if } 1 \leqslant x \leqslant t+r+1 \\
\left(\begin{array}{c}
p t+p-1 \\
p-1
\end{array}\right)-\left(\begin{array}{c}
p t+p-t-2+x \\
p-1
\end{array}\right), & \text { if } t+r+2 \leqslant x \leqslant t
\end{array}\right.
$$

As $p \rightarrow \infty$, the proportion of paths in $\Omega_{p, r}$, where $-t \leqslant r \leqslant-1$, with downcrossing number $x$ about $r+1$ is $X_{p, r}(r+1, x) B_{p, r}^{-1}$. As $p \rightarrow \infty$, this proportion, after some simplification using Stirling's formula, equals

$$
\begin{cases}\frac{1}{t}\left(\frac{1+t}{t}\right)^{-r-1}, & \text { if } 1 \leqslant x \leqslant t+r+1 \\ \frac{1}{t}\left(\frac{1+t}{t}\right)^{-r-1}-\frac{1}{t}\left(\frac{1+t}{t}\right)^{x-r-t-2}, & \text { if } t+r+2 \leqslant x \leqslant t .\end{cases}
$$

Acknowledgment. We would like to thank the referee for the helpful comments and for pointing out equation (19).

\section{References}

[1] Chen, R., Zame, A., Odlyzko, A., and Shepp, L., An optimal acceptance policy for an urn scheme, SIAM J. Discrete Math., Vol. 11, No. 2, 183-195, 1998.

[2] Feller, W., An Introduction to Probability Theory and its Applications, Vol. 1, 3rd edition, Wiley, 1968.

[3] Goulden, I., and Serrano, L., Maintaining the spirit of the reflection principle when the boundary has arbitrary integer slope, J. Combin. Theory, Ser. A, 104, 317-326, 2003.

[4] Graham, R., Knuth, D., and Patashnik, O., Concrete Mathematics: A Foundation for Computer Science, $2^{\text {nd }}$ edition, Addison-Wesley, 1994.

[5] Krattenthaler, C., The enumration of lattice paths with respect to their number of turns, in: N. Balakrishnan (Ed.), Advances in Cominatorial Methods and Applications to Probability and Statistics, Birkhaüuser, Boston, 29-58, 1997.

[6] Mohanty, S.G., On some generalization of a restricted random walk, Studia Sci. Math. Hungar., 3, 225-241, 1968.

[7] Mohanty, S.G., Lattice path Counting and Applications, Academic Press, New York, 1979.

[8] Niederhausen, H., How many paths cross at least $\ell$ given lattice points?, Congr. Numer., 36, 161-173, 1982.

[9] Renault, M., Four proofs of the ballot theorem, Math. Mag., 80, 345-352, 2007.

[10] Saran, J. and Rani, S., Rank Order Statistics Related to a Generalized Random Walk, in: N. Balakrishnan (Ed.), Advances in Combinatorial Methods and Applications to Probability and Statistics, Birkhaüuser, Boston, 135-151, 1997.

[11] Stanley, R.P., Enumerative Combinatorics, Vol 2, Cambridge University Press, 1997. 
[12] Suen, S. and Wagner K., Some Results for the Acceptance Urn Model, SIAM J. Discrete Math., 24, 876-891, 2010.

[13] Takács, L., On the ballot problem, in: N. Balakrishnan (Ed.), Advances in Combinatorial Methods and Applications to Probability and Statistics, Birkhäuser, Boston, 97-114, 1997. 